\title{
A no-go result on common cause approaches via Hardy relations
}

\author{
Katsuaki Higashi
}

\begin{abstract}
According to a conventional view, there exists no common cause model of quantum correlations satisfying locality requirements. Indeed, Bell's inequality is derived from some locality requirements and the assumption that the common cause exists, and the violation of the inequality has been experimentally verified. On the other hand, some researchers argued that in the derivation of the inequality, the existence of a common common-cause for multiple correlations is implicitly assumed and that the assumption is unreasonably strong. According to their idea, what is necessary for explaining the quantum correlation is a common cause for each correlation. However, Graßhoff et al. showed that when there are three pairs of perfectly correlated events and a common cause of each correlation exist, we cannot construct a common cause model that is consistent with quantum mechanical prediction and also meets several locality requirements. In this paper, first, as a consequence of the fact shown by Graßhoff et al., we will confirm that there exists no local common cause model when a two-particle system is in any maximally entangled state. After that, based on Hardy's famous argument, we will prove that there exists no local common cause model when a two-particle system is in any non-maximally entangled state. Therefore, it will be concluded that for any entangled state, there exists no local common cause model. It will be revealed that the non-existence of a common cause model satisfying locality is not limited to a particular state like the singlet state.
\end{abstract}

\section{Introduction}

A quantum correlation is a correlation between measurement results for each particle of a composite system composed of quantum mechanical objects (e.g. electrons). As is well known, the correlation can occur in two spatially separated regions. If, as in an orthodoxy, we do not acknowledge the existence of direct causal connections between two events which occur in such regions, then it is a natural idea that there exists a common cause of such correlated events.

In usual discussions concerning the question of whether there exists a common cause of a quantum correlation, as a result of the mathematical arguments, it is concluded that there exists no common cause model consistent with quantum mechanical predictions. However, some researchers argue that a disputable 
requirement is tacitly assumed in such discussions. The requirement is that there exists a common common-cause of multiple quantum correlations. According to their idea, what is necessary for explaining quantum correlations is a common cause for each correlation. In this paper, we discuss the possibility and impossibility of constructing a common cause model explaining quantum mechanical correlations.

There is no doubt about the importance of this theme. If we can construct a common cause model that satisfies locality requirements, we will have a new possibility to have a picture of how the quantum mechanical object behaves behind phenomena. On the other hand, if such a model does not exist, we must explore another way to understand quantum world.

\section{Backgrounds}

\subsection{Surface probability space}

In this section, we introduce a classical probability space consisting of (i) propositions representing measurement apparatus settings and measured effects and (ii) a probability measure on them. Van Fraassen (1982) called the phenomenological probabilities as surface probabilities. We use his terminology and notation.

Suppose spin measurements are performed for a composite system of two spin-1/2 particles (particle L and particle R). And suppose that by the measuring apparatus for particle $\mathrm{L}$, one of two specific incompatible spin observables will be measured. In other words, the apparatus $\mathrm{L}$ has two measurement settings $L_{i}(i=1,2)$. Likewise, the apparatus $\mathrm{R}$ has two measurement settings $R_{j}(j=1,2)$. For simplicity, it is assumed that in each experiment either one of the two spin observables is necessarily measured for each of the particles $\mathrm{L}$ and $\mathrm{R}$. In short, the apparatuses L and $\mathrm{R}$ have the following 4 possible measurement settings:

$$
\left\{L_{i} \wedge R_{j}: i, j=1,2\right\} .
$$

Here also, for simplicity, we will assume that in any measurement, either measured value + or - can always be obtained without errors. Then, in each measurement setting $L_{i} \wedge R_{j}$, there are the 4 possible results $L_{i a} \wedge R_{j b}(a=$ $+,-; b=+,-)$. The set of all possible measurement outcomes is as follows:

$$
\left\{L_{i a} \wedge R_{j b}: i, j=1,2 ; a, b=+,-\right\}
$$

It has 16 members. We can construct the Boolean algebra having these 16 measurement results as atoms. Below, we denote by $\mathcal{B}$ this Boolean algebra. $\mathcal{B}$ represents the propositional structure of the measured effects.

In the Boolean algebra $\mathcal{B}$, as we can see from how to construct the probability space, the following relation holds.

$$
L_{i} \wedge R_{j}=\left(L_{i+} \wedge R_{j+}\right) \vee\left(L_{i+} \wedge R_{j-}\right) \vee\left(L_{i-} \wedge R_{j+}\right) \vee\left(L_{i-} \wedge R_{j-}\right)
$$


An arbitrary proposition $X$ for measurement on one side (e.g. particle L) is defined as $X \equiv X \wedge\left(R_{1} \vee R_{2}\right)$. For example, $L_{1}$ and $L_{1+}$ are defined as $L_{1} \equiv$ $L_{1} \wedge\left(R_{1} \vee R_{2}\right)$ and $L_{1+} \equiv L_{1+} \wedge\left(R_{1} \vee R_{2}\right)$, respectively. From this definition and the propositional structure of $\mathcal{B}$, we can see that the following relation holds.

$$
L_{i}=L_{i+} \vee L_{i-}(i=1,2) ; R_{j}=R_{j+} \vee R_{j-}(j=1,2) .
$$

Next, we will define a probability measure on the Boolean algebra $\mathcal{B}$. Let $Q\left(L_{i a} \wedge R_{j b}\right)$ be the quantum mechanical prediction for a measurement result $L_{i a} \wedge R_{j b}$. According to Quantum mechanics, $Q\left(L_{i a} \wedge R_{j b}\right)=\operatorname{Tr}\left(D\left(P_{L_{i a}} \otimes\right.\right.$ $\left.P_{R_{j b}}\right)$ ), where $D$ denotes the density operator of the measured system and $P_{L_{i a}}$ $\left(P_{R_{j b}}\right)$ denotes the projection operator corresponding to the measured effect $L_{i a}$ $\left(R_{j b}\right)$. In order to be consistent with our experience, the probability measure $\operatorname{Pr}(\cdot)$ to be introduced must satisfy the following relations:

$$
\begin{aligned}
\operatorname{Pr}\left(L_{i a} \wedge R_{j b} \mid L_{i} \wedge R_{j}\right) & =Q\left(L_{i a} \wedge R_{j b}\right), \\
\operatorname{Pr}\left(L_{i a} \mid L_{i} \wedge R_{j}\right) & =Q\left(L_{i a}\right), \\
\operatorname{Pr}\left(R_{j b} \mid L_{i} \wedge R_{j}\right) & =Q\left(R_{j b}\right) .
\end{aligned}
$$

There are other relations that $\operatorname{Pr}(\cdot)$ should satisfy. Let $\mu\left(L_{i} \wedge R_{j}\right)$ denote a ratio at which the apparatuses $\mathrm{L}$ and $\mathrm{R}$ take a measurement setting $L_{i} \wedge R_{j}$. As already mentioned, the apparatuses $\mathrm{L}$ and $\mathrm{R}$ have the 4 possible settings $\left\{L_{i} \wedge R_{j}: i, j=1,2\right\}$. Therefore, $\sum_{i, j} \mu\left(L_{i} \wedge R_{j}\right)=1$. For the setting of the measuring device, the probability measure $\operatorname{Pr}(\cdot)$ must satisfy the following relations:

$$
\begin{gathered}
\operatorname{Pr}\left(L_{i} \wedge R_{j}\right)=\mu\left(L_{i} \wedge R_{j}\right), \\
\operatorname{Pr}\left(L_{i}\right)=\sum_{j} \mu\left(L_{i} \wedge R_{j}\right), \\
\operatorname{Pr}\left(R_{j}\right)=\sum_{i} \mu\left(L_{i} \wedge R_{j}\right) .
\end{gathered}
$$

So as to satisfy the relations (1) to (6), we define the probability measure $\operatorname{Pr}(\cdot)$ on the Boolean algebra $\mathcal{B}$ in the following way. First, for each atom of the Boolean algebra, we define the probability as follows:

$$
\operatorname{Pr}\left(L_{i a} \wedge R_{j b}\right) \equiv Q\left(L_{i a} \wedge R_{j b}\right) \mu\left(L_{i} \wedge R_{j}\right) .
$$

As can be easily seen, the sum of the values assigned to the 16 atoms of $\mathcal{B}$ is 1 . We can extend this definition to the whole algebra to satisfy additivity. Then, we can verify that the probability measure thus defined satisfies all the above relations. We call the classical probability space $(\mathcal{B}, P r)$ constructed in this way surface probability space.

From the above explanation, it is obvious that the following fact holds.

Fact 1. Suppose spin measurements are performed for a composite system of two spin-1/2 particles. For any two settings of each apparatus, and for any spin state, we can construct the surface probability space. 
In this fact, we supposed that each apparatus has two measurement settings. However, even if the number of measurement settings is any finite, we can construct the surface probability space in a similar way (for a mathematically rigorous proof, see Bana and Durt 1997).

\subsection{Conditions used to derive Bell's inequality}

In the surface probability space, a quantum mechanical correlation is represented as follows:

$$
\operatorname{Corr}\left(L_{i a}, R_{j b}\right)=P\left(L_{i a} \wedge R_{j b} \mid L_{i} \wedge R_{j}\right)-P\left(L_{i a} \mid L_{i} \wedge R_{j}\right) \cdot P\left(R_{j b} \mid L_{i} \wedge R_{j}\right) .
$$

If $\operatorname{Corr}\left(L_{i a}, R_{j b}\right) \neq 0$, then we say that $L_{i a}$ and $R_{j b}$ are correlated. In particular, when $P\left(L_{i a} \mid R_{j b} \wedge L_{i} \wedge R_{j}\right)=1$ and $P\left(R_{j b} \mid L_{i a} \wedge L_{i} \wedge R_{j}\right)=1$ hold, we say that $L_{i a}$ and $R_{j b}$ are perfectly correlated.

When a two-particle system is in an entangled state, a quantum mechanical correlation occurs by choosing spin components appropriately. Since a quantum mechanical correlation can occur in spatially separated regions, it is unlikely that direct causal connection exists between the correlated events. Then, it is natural to think that there exists a common cause for the two correlated events and that the correlation was caused by the common cause. Therefore, the following problem will be considered below. Is it possible to extend ${ }^{1}$ the surface probability space $(\mathcal{B}, P r)$ to a probability space that includes a common cause of a correlation that meets some locality requirements? In the following, we denote a probability measure in an extension of the surface probability space as $P$.

First, with respect to attempts to explain quantum mechanical correlations by a common causal explanation, the following mathematical result is well known (e.g. Bub 1997, and Redhead 1987).

Fact 2. Let $\left\{C_{k}\right\}_{k \in K}$ be a family of events satisfying the following conditions: (1) if $i \neq j(i, j \in K)$, then $C_{i} \wedge C_{j}=\emptyset$, (2) $P\left(C_{k}\right) \neq 0$ for any $k \in K$, and (3) $\sum_{k} P\left(C_{k}\right)=1$. Assuming each event belonging to $\left\{C_{k}\right\}_{k \in K}$ satisfies the following conditions, Bell's inequality is derived. ${ }^{2}$

A1 $P\left(L_{i a} \wedge R_{j b} \mid L_{i} \wedge R_{j} \wedge C_{k}\right)=P\left(L_{i a} \mid L_{i} \wedge R_{j} \wedge C_{k}\right) P\left(R_{j b} \mid L_{i} \wedge R_{j} \wedge C_{k}\right)$.

A2.1 $P\left(L_{i a} \mid L_{i} \wedge R_{j} \wedge C_{k}\right)=P\left(L_{i a} \mid L_{i} \wedge C_{k}\right)$.

A2.2 $P\left(R_{j b} \mid L_{i} \wedge R_{j} \wedge C_{k}\right)=P\left(R_{j b} \mid R_{j} \wedge C_{k}\right)$.

A3 $P\left(C_{k} \mid L_{i} \wedge R_{j}\right)=P\left(C_{k}\right)$.

\footnotetext{
${ }^{1}$ A classical probability space $\left(\mathcal{B}^{\prime}, P^{\prime}\right)$ (where $\mathcal{B}^{\prime}$ a Boolean algebra and $P^{\prime}$ is a probability measure on it) is called an extension of $(\mathcal{B}, P)$ (where $\mathcal{B}$ a Boolean algebra and $P$ is a probability measure on it), if there exists a Boolean homomorphism $h: \mathcal{B} \rightarrow \mathcal{B}^{\prime}$ such that $P^{\prime}(h(X))=P(X)$ for all $X \in \mathcal{B}$.

${ }^{2}$ In this paper, we discuss only when $\left\{C_{k}\right\}_{k \in K}$ is a countable set. If we change slightly the notation, the same conclusion is obtained even when the parameters are continuous.
} 
The reasons for imposing the respective conditions are as follows. If $\left\{C_{k}\right\}_{k \in K}$ is a set of events satisfying the condition A1, the quantum mechanical correlation disappears when an event belonging to the set occurs. Therefore, the events are considered as a candidate for the cause of the correlation.

The condition A2.1 is equivalent to the following condition: $P\left(L_{i a} \mid L_{i} \wedge R_{l} \wedge\right.$ $\left.C_{k}\right)=P\left(L_{i a} \mid L_{i} \wedge R_{m} \wedge C_{k}\right)(l \neq m)$. This condition says, once the cause of the correlation occurs, even if we change the setting of the apparatus $\mathrm{R}$ (immediately before the measurement for particle L), the probability of the measured result of particle $\mathrm{L}$ will not change. The same is true for the condition A2.2.

A3 only requires that each event of $\left\{C_{k}\right\}_{k \in K}$ is statistically independent of the setting of the measuring device. However actually, when this condition is satisfied, any event constructed from $\left\{C_{k}\right\}_{k \in K}$ (e.g. $C_{i} \vee C_{j}, C_{i}^{\perp}$ ) is statistically independent of the setting of the measuring device. Since $C_{k}$ is a candidate for a common cause of a correlation between measurement results that can occur in two spatially separated regions, it is natural to think that $C_{k}$ occurs in the overlap of backward light cones of each spatiotemporal region. Considering that we can change the device settings even after $C_{k}$ occurred, it is reasonable to require the condition A3.

Bell's inequality has been verified experimentally and its violation has been confirmed (e.g. Aspect, Dalibard, and Roger 1982). When the inequality is violated, there exists no mathematical model that satisfies all the above requirements.

\subsection{Szabó's common cause model and a no-go result}

Hofer-Szabó, Rédei, and Szabó (1999) pointed out that the derivation of Bell's inequality implicitly presupposes the existence of a common common-cause for multiple correlations. Originally, the principle of the common cause is merely requiring the existence of a common cause for each correlation (see Reichenbach 1956). Nevertheless, in the derivation of Bell's inequality, the single family of events $\left\{C_{k}\right\}_{k \in K}$ is considered as the common cause of the multiple correlations. According to their idea, it is sufficient that each quantum correlation $\operatorname{Corr}\left(L_{i a}, R_{j b}\right) \neq 0$ has respectively the common cause events $\left\{C_{k}^{i j}\right\}_{k \in K_{i j}}$. Therefore, we define the events (we call them screening-off factors) which are candidates for common causes of each correlation as follows.

Definition 1. Suppose $L_{i a}$ and $R_{j b}$ are correlated (i.e. $\operatorname{Corr}\left(L_{i a}, R_{j b}\right) \neq 0$ ). We call the events $\left\{C_{k}^{i j}\right\}_{k \in K_{i j}}$ which satisfy the following conditions screeningoff factors of $\operatorname{Corr}\left(L_{i a}, R_{j b}\right)$.

(a) If $l \neq m\left(l, m \in K_{i j}\right)$, then $C_{l}^{i j} \wedge C_{m}^{i j}=\emptyset$.

(b) $P\left(C_{k}^{i j}\right) \neq 0$ for any $k \in K_{i j}$.

(c) $\sum_{k \in K_{i j}} P\left(C_{k}^{i j}\right)=1$.

(d) $P\left(L_{i a} \wedge R_{j b} \mid L_{i} \wedge R_{j} \wedge C_{k}^{i j}\right)=P\left(L_{i a} \mid L_{i} \wedge R_{j} \wedge C_{k}^{i j}\right) \cdot P\left(R_{j b} \mid L_{i} \wedge R_{j} \wedge C_{k}^{i j}\right)$. 
Note the following about this definition. In the notation of the screening-off factors $\left\{C_{k}^{i j}\right\}_{k \in K_{i j}}$ of the correlation $\operatorname{Corr}\left(L_{i a}, R_{j b}\right), a$ and $b$ do not appear. As can be easily ascertained, if there exist screening-off factors of one correlation (e.g. $\operatorname{Corr}\left(L_{i+}, R_{j+}\right)$ ), they are also screening-off factors of the other correlations $\left(\operatorname{Corr}\left(L_{i+}, R_{j-}\right), \operatorname{Corr}\left(L_{i-}, R_{j+}\right)\right.$, and $\left.\operatorname{Corr}\left(L_{i-}, R_{j-}\right)\right)$. Therefore, we do not need to specify a value of $a$ and $b$ in the notation of the screening-off factors.

As Hofer-Szabó et al. proposed, in common causal explanation of multiple quantum correlations, is it truly sufficient if only the common cause of each correlation exists? This is a controversial issue.

On the one hand, the following mathematical fact that questions this idea is well known (e.g. Uffink(1999)). Even when $C$ screens off the correlation between $X$ and $Y$ (i.e. $P(X \wedge Y \mid C)=P(X \mid C) \cdot P(Y \mid C))$, there may well exist a further event $E$ in the common past of $X$ and $Y$ such that $P(X \wedge Y \mid C \wedge E) \neq$ $P(X \mid C \wedge E) \cdot P(Y \mid C \wedge E)$. For example, $E$ may be a screening-off factor of other correlation. Even if $C$ screens off the correlation between $X$ and $Y$, if there still remains a correlation between $X$ and $Y$ under $C \wedge E$, then it will be necessary to explain this conditional correlation newly. Such a demand to the explanation ends only if there exist common screening-off factors for all correlations.

On the other hand, it is interesting that common screening-off factors maybe not always exist in general, regardless of quantum correlations. Hofer-Szabó, Rédei, and Szabó (2002) showed the following fact: when the cardinality of the index set of screening-off factors is limited to 2 (i.e. a common cause in Reichenbach's original sense), a common cause of each correlation always exists, however, in some case, common common-cause of multiple correlations does not exist. If it will be proved without the restriction on the index set that there cannot exist common screening-off factors of multiple correlations in some case, it will be very interesting. In that case, regardless of quantum mechanics, common screening-off factors do not always exist, therefore, requiring common screening-off factors of multiple correlations is maybe unreasonably too strong.

It is very interesting to develop these conceptual arguments. However, in this paper, we focus on the mathematical possibilities and impossibility of the common cause model and proceed with the discussion.

Szabó $(1998,2000)$ constructed a mathematical model that contains screeningoff factors of each correlation and also that satisfies some conditions related to locality.

Fact 3. (Szabó) Suppose spin measurements are performed for a composite system of two spin- $1 / 2$ particles. For any two settings of each apparatus, and for any spin state, there exists an extension of the surface probability space which contains screening-off factors $\left\{C_{k}^{i j}\right\}_{k \in K}(i, j=1,2)$ of each correlation which satisfy the following conditions:

B1.1 $P\left(L_{i a} \mid L_{i} \wedge R_{j} \wedge C_{k}^{i j}\right)=P\left(L_{i a} \mid L_{i} \wedge C_{k}^{i j}\right)$.

B1.2 $P\left(R_{j b} \mid L_{i} \wedge R_{j} \wedge C_{k}^{i j}\right)=P\left(R_{j b} \mid R_{j} \wedge C_{k}^{i j}\right)$. 
B2 $P\left(C_{k}^{i j} \mid L_{i} \wedge R_{j}\right)=P\left(C_{k}^{i j}\right){ }^{3}$

Although this is a remarkable fact, it is still questionable whether this model is truly local. Actually, as Szabó himself pointed out (see "postscript" in Szabó 1998), his model does not satisfy the following condition which is stronger than B2.

$\mathcal{C}$-independence: Let $\mathcal{C}$ be the Boolean subalgebra generated by $\left\{C_{k}^{11}\right\}_{k \in K} \cup$ $\left\{C_{l}^{12}\right\}_{l \in L} \cup\left\{C_{m}^{21}\right\}_{m \in M} \cup\left\{C_{n}^{22}\right\}_{n \in N}$. Then, for any atom $Z$ of $\mathcal{C}$,

$$
P\left(Z \mid L_{i} \wedge R_{j}\right)=P(Z) \quad(i, j=1,2) .
$$

The difference between $\mathrm{B} 2$ and $\mathcal{C}$-independence is as follows. In B2, the index of the measurement-setting is identical to that of the screening-off factor. Therefore, according to this condition, for example, $L_{1} \wedge R_{1}$ is required to be statistically independent of $C_{k}^{11}$, however, it is not always statistically independent of $C_{k}^{12}$ and $C_{k}^{22}$. On the other hand, when satisfying $\mathcal{C}$-independence, any element belonging to $\mathcal{C}$ is statistically independent of any measurement setting.

The reason for imposing $\mathcal{C}$-independence is as follows. As mentioned above, it is natural to think that a common cause occurs in the overlap of the past light cones of the two spatially separated regions where the measurements are made. Also, for each correlation $\operatorname{Corr}\left(L_{i a}, R_{j b}\right)$, one event belonging to $\left\{C_{k}^{i j}\right\}_{k \in K}$ must occur because $\sum_{k} P\left(C_{k}^{i j}\right)=1$. As this is true for all correlations, any one of the atoms of $\mathcal{C}$ must occur in the overlap of the two past light cones. However, even after such an event occurred, we can change the apparatus setting. Therefore, $\mathcal{C}$-independence is a requirement to be satisfied.

Although Szabó's model does not satisfy $\mathcal{C}$-independence, there may exist other models that satisfy all the above requirements. However, the following negative result is known.

Fact 4. (Graßhoff, Portmann, and Wüthrich 2005) Suppose spin measurements are performed for a composite system of two spin-1/2 particles. Furthermore, suppose the following hold.

1. Three spin components are measurable on each side, i.e. $L_{i}(i=1,2,3)$ and $R_{j}(j=1,2,3)$.

2. For any $i \in\{1,2,3\}, L_{i+}$ and $R_{i-}$ are perfectly correlated.

When there exist screening-off factors of each perfect correlation, and they satisfy $B 1$ and $\mathcal{C}$-independence, Bell's inequality (Wigner form) :

$$
P\left(L_{1+} \wedge R_{3+} \mid L_{1} \wedge R_{3}\right) \leq P\left(L_{1+} \wedge R_{2+} \mid L_{1} \wedge R_{2}\right)+P\left(L_{2+} \wedge R_{3+} \mid L_{2} \wedge R_{3}\right)
$$

\footnotetext{
${ }^{3}$ B1, B2, and (d) in Def.1 are different from the conditions Szabó $(1998,2000)$ explicitly stated in his paper. However, as can be seen from (11) and (25) in his paper in 1998, his model satisfies the following four relations: (a) $P\left(L_{i} \wedge R_{j}\right)=P\left(L_{i}\right) \cdot P\left(R_{j}\right)$, (b) $P\left(C_{k}^{i j} \wedge L_{i} \wedge R_{j}\right)=$ $P\left(C_{k}^{i j}\right) \cdot P\left(L_{i} \wedge R_{j}\right),\left(\right.$ c) $P\left(L_{i a} \wedge R_{j} \wedge C_{k}^{i j}\right)=P\left(L_{i a} \wedge C_{k}^{i j}\right) \cdot P\left(R_{j}\right) ; P\left(L_{i} \wedge R_{i b} \wedge C_{k}^{i j}\right)=$ $P\left(R_{j b} \wedge C_{k}^{i j}\right) \cdot P\left(L_{i}\right)$, (d) $P\left(L_{i a} \wedge R_{j b} \mid C_{k}^{i j}\right)=P\left(L_{i a} \mid C_{k}^{i j}\right) \cdot P\left(R_{j b} \mid C_{k}^{i j}\right)$. Then, we can easily show that his model satisfies our conditions.
} 
is derived. ${ }^{4}$

Fact 4 was shown using the following well-known fact: when $X$ and $Y$ are perfectly correlated, a determinism holds for any screening-off factor $C_{i}$ of the correlation, namely $P\left(X \mid C_{i}\right)$ and $P\left(Y \mid C_{i}\right)$ are 0 or 1 . When the determinism is established for screening-off factors of each correlation, we can construct common screening-off factors for multiple correlations, then Bell's inequality is derived. For example, when a composite system is in the singlet state and three pairs of identical spin components are measurable (i.e. three parallel settings), a perfect correlation occurs between the measurement results of each of the three pairs, and as a result, the above inequality is derived. When the inequality is violated, there exists no common cause model which satisfies all of the above requirements and is consistent with quantum mechanical predictions.

Fact 4 was shown in the specific situation (i.e. perfect correlation). Later, Portmann and Wüthrich (2007) and Hofer-Szabó (2011) independently showed that an inequality which is inconsistent with quantum mechanical prediction is derived without assuming perfect correlations. More specifically, they derived the inequality under the situation that there exist multiple pairs that are almost perfectly correlated. It is clear that their proofs are valid when the composite system is in the singlet state. However, I did not know how their proofs can be applied when the system is in one of the other states, especially when the system is in an entangled state other than the maximally entangled states. ${ }^{5}$ Because it is not trivial that there exist multiple pairs that are almost perfectly correlated when the system is in such a state.

\section{A no-go result for all entangled states}

As stated in Sect. 2.3, when a composite system is in the singlet state, we cannot give the common causal explanation of quantum correlations. Is the singlet state the only specific state that we cannot give such an explanation? It is not. In fact, as we will show, it is impossible to construct a common cause model of quantum correlations for any entangled state.

We will classify the quantum entangled states into two types (the maximally entangled states and the other entangled states) and prove that. In order to classify the states, we will use the following famous mathematical fact (Schmidt decomposition theorem ${ }^{6}$ ). The theorem holds for any pure state on the tensor product space of any two finite-dimensional Hilbert spaces. However, since the following discussion uses only the 2-dimensional Hilbert space, we describe the mathematical fact only for 2-dimensional case.

Schmidt decomposition theorem Let $\mathcal{H}_{L}^{2}$ and $\mathcal{H}_{R}^{2}$ be the (2-dimensional) spin state spaces of the particle $\mathrm{L}$ and the particle $\mathrm{R}$, respectively. Then, for any

\footnotetext{
${ }^{4}$ See Wigner (1970).

${ }^{5}$ For the definition of maximally entangled states, see the next section.

${ }^{6}$ For proof, see e.g. Nielsen and Chuang (2000).
} 
pure state $|\Phi\rangle$ of the composite system (particles L and R), there exists an orthonormal basis $\left\{\left|e_{1}\right\rangle_{L},\left|e_{2}\right\rangle_{L}\right\}$ for $\mathcal{H}_{L}^{2}$, and an orthonormal basis $\left\{\left|f_{1}\right\rangle_{R},\left|f_{2}\right\rangle_{R}\right\}$ for $\mathcal{H}_{R}^{2}$ such that

$$
|\Phi\rangle=\alpha\left|e_{1}\right\rangle_{L} \otimes\left|f_{1}\right\rangle_{R}+\beta\left|e_{2}\right\rangle_{L} \otimes\left|f_{2}\right\rangle_{R}
$$

where $\alpha, \beta$ are non-negative real numbers satisfying $\alpha^{2}+\beta^{2}=1$ known as Schmidt coefficients.

Schmidt decomposition is unique (ignoring the phase factor) if and only if all Schmidt coefficients are distinct (i.e. $\alpha \neq \beta$ in 2-dimensional case). When more than one Schmidt coefficients of a state are non-zero (i.e. $\alpha, \beta \neq 0$ in 2-dimensional case), we call the state an entangled state. In particular, when all Schmidt coefficients are identical (i.e. $\alpha=\beta$ in 2-dimensional case), it is called a maximally entangled state. For example, the singlet state is one of the maximally entangled states. In addition, we call entangled states other than the maximally entangled states non-maximally entangled states.

Below, first, we will confirm that the common cause model does not exist when the composite system is in a maximally entangled state (Fact 5). This is a consequence of Fact 4 in Sect. 2.3. And then we will show that a similar fact holds in all non-maximally entangled states. To show the fact, we will use Hardy's famous argument.

Many of the previous studies discussing the possibility and impossibility of a common cause model have been paying attention to whether Bell's inequality is derived or not. However, the important is consistency with quantum mechanical predictions. There are two virtues that arise from using Hardy's argument. One is that the impossibility of common cause model will be shown in many physical states. The other is that the impossibility proof is quite simple.

\subsection{Maximally entangled states}

From Fact 4 it is clear that there exists no common cause model that satisfies B1 and $\mathcal{C}$-independence when a composite system is in the singlet state. However, there exist also maximally entangled states other than the singlet state. In this section, using Fact 4, we will show that for any maximally entangled state, there exists no common cause model that satisfies B1 and $\mathcal{C}$-independence.

When a composite system is in a maximally entangled state, the Schmidt decomposition of the state is as follows:

$$
|\Omega\rangle=\frac{1}{\sqrt{2}}\left|e_{1}\right\rangle_{L} \otimes\left|f_{1}\right\rangle_{R}+\frac{1}{\sqrt{2}}\left|e_{2}\right\rangle_{L} \otimes\left|f_{2}\right\rangle_{R} .
$$

Using three distinct real numbers $0 \leq \eta_{1}, \eta_{2}, \eta_{3} \leq \pi$, we define the unit vectors $\left|p_{i}\right\rangle_{L} \in \mathcal{H}_{L}^{2}$ and $\left|q_{j}\right\rangle_{R} \in \mathcal{H}_{R}^{2}$ respectively as follows:

$$
\begin{gathered}
\left|p_{i}\right\rangle_{L} \equiv \cos \eta_{i}\left|e_{1}\right\rangle_{L}+\sin \eta_{i}\left|e_{2}\right\rangle_{L} \quad(i=1,2,3), \\
\left|q_{j}\right\rangle_{R} \equiv \sin \eta_{j}\left|f_{1}\right\rangle_{R}-\cos \eta_{j}\left|f_{2}\right\rangle_{R} \quad(j=1,2,3) .
\end{gathered}
$$


Furthermore, using these unit vectors, we define the following projection operators on $\mathcal{H}_{L}^{2}$ and $\mathcal{H}_{R}^{2}$ :

$$
P_{i} \equiv\left|p_{i}\right\rangle_{L}\left\langle\left. p_{i}\right|_{L} \quad(i=1,2,3), \quad Q_{j} \equiv \mid q_{j}\right\rangle_{R}\left\langle\left. q_{j}\right|_{R} \quad(j=1,2,3) .\right.
$$

Then, as a result of easy calculation, the following relation holds.

$$
Q^{|\Omega\rangle}\left(P_{i} \wedge Q_{j}\right)=\frac{1}{2} \sin ^{2}\left(\eta_{j}-\eta_{i}\right) .
$$

We will use the following notation.

$L_{i}(i=1,2,3)$ : the apparatus $\mathrm{L}$ is set to measure $P_{i}$.

$L_{i+}(i=1,2,3)$ : the result of $P_{i}$-measurement is +1 .

$R_{j}(j=1,2,3)$ : the apparatus $\mathrm{L}$ is set to measure $Q_{j}$.

$R_{j+}(j=1,2,3)$ : the result of $Q_{j}$-measurement is +1 .

In the surface probability space including these events, the quantum mechanical correlation $Q^{|\Omega\rangle}\left(P_{i} \wedge Q_{j}\right)$ is represented as follows:

$$
P\left(L_{i+} \wedge R_{j+} \mid L_{i} \wedge R_{j}\right)=\frac{1}{2} \sin ^{2}\left(\eta_{j}-\eta_{i}\right) .
$$

In particular, when $i=j, P\left(L_{i+} \wedge R_{i+} \mid L_{i} \wedge R_{i}\right)$ is 0 , therefore $L_{i+}$ and $R_{i+}$ are perfectly anti-correlated. In other words, $L_{i+}$ and $R_{i-}$ are perfectly correlated $(i=1,2,3)$. Then, Bell's inequality (Wigner form) is derived from Fact 4 . For example, when $\eta_{1}=\frac{\pi}{3}, \eta_{2}=\frac{\pi}{2}$, and $\eta_{3}=\frac{2 \pi}{3}$, the probabilities are as follows:

$$
\begin{aligned}
& P\left(L_{1+} \wedge R_{3+} \mid L_{1} \wedge R_{3}\right)=\frac{3}{8}, \\
& P\left(L_{1+} \wedge R_{2+} \mid L_{1} \wedge R_{2}\right)=\frac{1}{8}, \\
& P\left(L_{2+} \wedge R_{3+} \mid L_{2} \wedge R_{3}\right)=\frac{1}{8} .
\end{aligned}
$$

In this case, it becomes $\frac{3}{8}>\frac{1}{8}+\frac{1}{8}$, and the inequality is violated. Therefore, the following facts hold.

Fact 5. For any maximally entangled states, there exists no extension from the surface probability space to a classical probability space which includes screeningoff factors of each correlation satisfying $B 1$ and $\mathcal{C}$-independence.

\section{$3.2 \quad$ Non-maximally entangled states}

In this section, we will show that for any non-maximally entangled state, there exists no common cause model which satisfies B1 and $\mathcal{C}$-independence and is consistent with quantum mechanical predictions. We will prove this fact by using four relations which appear in Hardy's famous argument (Hardy 1993). 


\subsubsection{Hardy's argument}

Let us review briefly Hardy's argument. First, we define the unit vectors $|a\rangle_{L},|b\rangle_{L}$ on $\mathcal{H}_{L}^{2}$ by using the Schmidt coefficients as follows:

$$
\begin{gathered}
|a\rangle_{L} \equiv \frac{1}{\sqrt{(1-\alpha \beta)(\alpha+\beta)}}\left(\beta \sqrt{\beta}|+\rangle_{L}-\alpha \sqrt{\alpha} i|-\rangle_{L}\right), \\
|b\rangle_{L} \equiv \sqrt{\frac{\beta}{\alpha+\beta}}|+\rangle_{L}+\sqrt{\frac{\alpha}{\alpha+\beta}} i|-\rangle_{L} .
\end{gathered}
$$

Also, we define the unit vectors $|a\rangle_{R}$ and $|b\rangle_{R}$ in the Hilbert space $\mathcal{H}_{R}^{2}$ in the same form. Next, using $\left|a_{L}\right\rangle$ and $|b\rangle_{L}$, we define the projection operators $A_{L}$ and $B_{L}$ on $\mathcal{H}_{L}^{2}$ as follows:

$$
A_{L} \equiv|a\rangle_{L}\left\langle\left. a\right|_{L}, B_{L} \equiv \mid b\right\rangle_{L}\left\langle\left. b\right|_{L} .\right.
$$

Also, we define the projection operators $A_{R}$ and $B_{R}$ on $\mathcal{H}_{R}^{2}$ in the same way.

Suppose that a composite system is in a non-maximally entangled state $|\Psi\rangle$. For the Schmidt coefficients $\alpha$ and $\beta$ of $|\Psi\rangle, \alpha, \beta \neq 0$ and $\alpha \neq \beta$. Then, we see that as a result of the elementary calculations, the quantum mechanical probabilities for $A_{L}, B_{L}, A_{R}, B_{R}$ satisfy the following relations:

C1 $Q^{|\Psi\rangle}\left(A_{L} \wedge A_{R}\right)=\frac{\alpha^{2} \beta^{2}(\alpha-\beta)^{2}}{(1-\alpha \beta)^{2}}>0$,

C2 $Q^{|\Psi\rangle}\left(B_{R} \mid A_{L}\right)=1$,

C3 $Q^{|\Psi\rangle}\left(B_{L} \mid A_{R}\right)=1$,

C4 $Q^{|\Psi\rangle}\left(B_{L} \wedge B_{R}\right)=0$.

In the following, we call these four relations Hardy relations.

Hardy's argument goes as follows. Suppose $A_{L} \wedge A_{R}$ is true (this is possible by $\mathrm{C} 1$ ). Then, using $\mathrm{C} 2$ and $\mathrm{C} 3, B_{L} \wedge B_{R}$ is true. However, this contradicts with $\mathrm{C} 4$. Here, note that if the state of a two-particles system is a non-entangled state (i.e. either $\alpha$ or $\beta$ is 0 ) or a maximally entangled state (i.e. $\alpha=\beta$ ), then the value of $\mathrm{C} 1$ is 0 , thus Hardy's argument does not hold.

\subsubsection{Preparation for derivation}

If the four events corresponding respectively to the four projections $A_{L}, B_{L}$, $A_{R}, B_{R}$ exist in the Boolean algebra of a classical probability space, and the quantum mechanical probabilities $Q^{|\Psi\rangle}(\cdot)$ are assigned to those events respectively, then, as explained in Sect. 3.2.1, Hardy's argument holds. However, in the surface probability space, quantum mechanical probabilities are represented as conditional probabilities. As mentioned in Fact 1, any measurement probabilities for $A_{L}, B_{L}, A_{R}, B_{R}$ are representable in the surface probability space. Indeed, as we will see, we can represent Hardy relations (C1-4) in the surface probability space. 
Let us use the following notation.

$L_{1}$ : the apparatus $\mathrm{L}$ is set to measure $A_{L}$.

$L_{1+}$ : the result of $A_{L}$-measurement is +1 .

$L_{2}$ : the apparatus $\mathrm{L}$ is set to measure $B_{L}$.

$L_{2+}$ : the result of $B_{L}$-measurement is +1 .

$R_{1}$ : the apparatus $\mathrm{L}$ is set to measure $A_{R}$.

$R_{1+}$ : the result of $A_{R}$-measurement is +1 .

$R_{2}$ : the apparatus $\mathrm{L}$ is set to measure $B_{R}$.

$R_{2+}$ : the result of $B_{R}$-measurement is +1 .

Then, Hardy relations (C1-4) are rewritten as follows:

D1 $P\left(L_{1+} \wedge R_{1+} \mid L_{1} \wedge R_{1}\right)=\frac{\alpha^{2} \beta^{2}(\alpha-\beta)^{2}}{(1-\alpha \beta)^{2}}>0$,

D2 $P\left(R_{2+} \mid L_{1+} \wedge L_{1} \wedge R_{2}\right)=1$,

D3 $P\left(L_{2+} \mid R_{1+} \wedge L_{2} \wedge R_{1}\right)=1$,

D4 $P\left(L_{2+} \wedge R_{2+} \mid L_{2} \wedge R_{2}\right)=0$.

First, let us confirm that $L_{1+}$ and $R_{2+}$ are positively correlated. We cannot derive this fact from D2 alone. In general, from $P(Y \mid X)=1$ and $P(Y)<1$, we can derive $P(X \wedge Y)-P(X) P(Y)>0$. Therefore, to derive the positive correlation, the relation $P\left(R_{2+} \mid L_{1} \wedge R_{2}\right)<1$ is further needed. By the calculation using the state $|\Psi\rangle$ and the projection operators $A_{L}, B_{R}$, we have $P\left(R_{2+} \mid L_{1} \wedge R_{2}\right)=\alpha \beta<1$. Thus, $L_{1+}$ and $R_{2+}$ are positively correlated (i.e. $\left.\operatorname{Corr}\left(L_{1+}, R_{2+}\right)>0\right)$. In a similar way, we can derive $\operatorname{Corr}\left(L_{2+}, R_{1+}\right)>0$ from D3 and $P\left(L_{2+} \mid L_{1} \wedge R_{2}\right)<1$.

Note that the correlations $\operatorname{Corr}\left(L_{1+}, R_{2+}\right)>0$ and $\operatorname{Corr}\left(L_{2+}, R_{1+}\right)>0$ cannot be perfect correlations. We can show this fact by direct calculation, or by reductio ad absurdum as follows. Suppose these events (e.g. $L_{1+}$ and $R_{2+}$ ) are perfectly correlated in a non-maximally entangled state. Then, in addition to the orthonormal basis used in Schmidt decomposition, there exists another orthonormal basis taking a bi-orthogonal form. However, that contradicts the uniqueness of Schmidt decomposition.

In Sect. 3.2.3, we will show there exists no common cause model of the positive correlations $\left(\operatorname{Corr}\left(L_{1+}, R_{2+}\right)>0\right.$ and $\left.\operatorname{Corr}\left(L_{2+}, R_{1+}\right)>0\right)$ which satisfies $\mathrm{B} 1$ and $\mathcal{C}$-independence. In its derivation, we will assume the following two as before.

Assumption $1 P\left(L_{i} \wedge R_{j}\right)>0(i, j=1,2)$.

Assumption $2 \sum_{i, j} P\left(L_{i} \wedge R_{j}\right)=1 \quad(i, j=1,2)$.

Also, in the derivation, we will use the following two lemmas. Since both can be proved easily, those proofs are omitted.

Lemma 1. Suppose $P(Y \mid X)=1$, then for any event $Z$, either $P(Y \mid X \wedge Z)=1$ or $P(X \wedge Z)=0$. 
Lemma 2. Suppose $P(Y \wedge Z) \neq 0$, then the following two expressions are equivalent.

- $P(X \wedge Y \mid Z)=P(X \mid Z) \cdot P(Y \mid Z)$.

- $P(X \mid Y \wedge Z)=P(X \mid Z)$.

\subsubsection{Derivation of a no-go result}

In this section, we will show the following fact.

Fact 6. For any non-maximally entangled state, there exists no extension from the surface probability space to a classical probability space which includes screeningoff factors of the positive correlations $\left(\operatorname{Corr}\left(L_{1+}, R_{2+}\right)>0\right.$ and $\operatorname{Corr}\left(L_{2+}, R_{1+}\right)>$ 0) satisfying $B 1$ and $\mathcal{C}$-independence.

Proof. Suppose there exist the screening-off factors $\left\{C_{k}^{12}\right\}_{k \in K}$ and $\left\{C_{l}^{21}\right\}_{l \in L}$ of the positive correlations $\operatorname{Corr}\left(L_{1+}, R_{2+}\right)>0$ and $\operatorname{Corr}\left(L_{2+}, R_{1+}\right)>0$ respectively, and these events satisfy B1 and $\mathcal{C}$-independence. We define the two events $C_{L_{1-}}, C_{R_{2+}}$ as follows:

$$
\begin{aligned}
& C_{L_{1-}} \equiv \vee\left\{C_{k}^{12}(k \in K): P\left(L_{1+} \wedge C_{k}^{12} \wedge L_{1} \wedge R_{2}\right)=0\right\}, \\
& C_{R_{2+}} \equiv \vee\left\{C_{k}^{12}(k \in K): P\left(L_{1+} \wedge C_{k}^{12} \wedge L_{1} \wedge R_{2}\right) \neq 0\right\} .
\end{aligned}
$$

Then, by these definitions and Definition 1 , we have $C_{L_{1-}} \wedge C_{R_{2+}}=\emptyset$ and $P\left(C_{L_{1-}} \vee C_{R_{2+}}\right)=1$. Therefore, for any event $X$,

$$
P\left(\neg C_{L_{1-}} \wedge X\right)=P\left(C_{R_{2+}} \wedge X\right) .
$$

Suppose $C_{k}^{12} \leq C_{L_{1-}}$. Then, $P\left(L_{1+} \mid C_{k}^{12} \wedge L_{1} \wedge R_{2}\right)=0 .{ }^{7}$ Applying B1.1 to this, $P\left(L_{1+} \mid C_{k}^{12} \wedge L_{1}\right)=0$, thus we have $P\left(L_{1+} \wedge C_{k}^{12} \wedge L_{1}\right)=0$. Since that holds for any $k$ satisfying $C_{k}^{12} \leq C_{L_{1-}}$, we have $P\left(L_{1+} \wedge C_{L 1-} \wedge L_{1}\right)=0$. By the propositional structure in the surface probability space, $L_{1+} \wedge L_{1}=L_{1+}$. Therefore, we have $P\left(L_{1+} \wedge C_{L 1-}\right)=0$, thus $P\left(C_{L_{1-}} \mid L_{1+}\right)=0$. That means $P\left(\neg C_{L_{1-}} \mid L_{1+}\right)=1$. Using (8), we have

$$
P\left(C_{R_{2+}} \mid L_{1+}\right)=1 .
$$

Suppose $C_{k}^{12} \leq C_{R_{2+}}$. Then, applying Lemma 1 to Hardy relation D2, we have $P\left(R_{2+} \mid L_{1+} \wedge C_{k}^{12} \wedge L_{1} \wedge R_{2}\right)=1$. Since $C_{k}^{12}$ is a screening-off factor of $\operatorname{Corr}\left(L_{1+}, R_{2+}\right)$, using Lemma 2, $P\left(R_{2+} \mid C_{k}^{12} \wedge L_{1} \wedge R_{2}\right)=1$. Furthermore, by B1.2, we have $P\left(R_{2+} \mid C_{k}^{12} \wedge R_{2}\right)=1$. Since that holds for any $k$ satisfying $C_{k}^{12} \leq C_{R_{2+}}$, we have

$$
P\left(R_{2+} \mid C_{R_{2+}} \wedge R_{2}\right)=1 .
$$

Similarly, we will define the two events $C_{R_{1-}}$ and $C_{L_{2+}}$.

$$
C_{R_{1-}} \equiv \vee\left\{C_{l}^{21}(l \in L): P\left(R_{1+} \wedge C_{l}^{21} \wedge L_{2} \wedge R_{1}\right)=0\right\} .
$$

\footnotetext{
${ }^{7}$ This conditional probability is definable because $P\left(C_{k}^{12} \wedge L_{1} \wedge R_{2}\right) \neq 0$ by Assumption 1 , Definition 1 (b), and $\mathcal{C}$-independence.
} 


$$
C_{L_{2+}} \equiv \vee\left\{C_{l}^{21}(l \in L): P\left(R_{1+} \wedge C_{l}^{21} \wedge L_{2} \wedge R_{1}\right) \neq 0\right\} .
$$

By a similar argument to the previous paragraph, we have

$$
\begin{gathered}
P\left(C_{L_{2+}} \mid R_{1+}\right)=1 . \\
P\left(L_{2+} \mid C_{L_{2+}} \wedge L_{2}\right)=1,
\end{gathered}
$$

Finally, a contradiction is derived as follows. By (9) and (11),

$$
P\left(C_{L_{2+}} \wedge C_{R_{2+}} \mid L_{1+} \wedge R_{1+}\right)=1 .
$$

(Note that $P\left(L_{1+} \wedge R_{1+}\right)>0$ because of Hardy relation D1.) By (13), we have

$$
P\left(C_{L_{2+}} \wedge C_{R_{2+}}\right)>0 .
$$

Applying Lemma 1 to (10), we have the following:

either $P\left(R_{2+} \mid C_{L_{2+}} \wedge C_{R_{2+}} \wedge L_{2} \wedge R_{2}\right)=1$ or $P\left(C_{L_{2+}} \wedge C_{R_{2+}} \wedge L_{2} \wedge R_{2}\right)=0$.

Also, by (14), Assumption 1, and $\mathcal{C}$-independence, we have

$$
P\left(C_{L_{2+}} \wedge C_{R_{2+}} \wedge L_{2} \wedge R_{2}\right) \neq 0 .
$$

Then, by (15) and (16),

$$
P\left(R_{2+} \mid C_{L_{2+}} \wedge C_{R_{2+}} \wedge L_{2} \wedge R_{2}\right)=1 .
$$

Applying Lemma 1 to (12), as a result of a similar argument to the previous paragraph, we have

$$
P\left(L_{2+} \mid C_{L_{2+}} \wedge C_{R_{2+}} \wedge L_{2} \wedge R_{2}\right)=1 .
$$

Then, by (17) and (18),

$$
P\left(L_{2+} \wedge R_{2+} \mid C_{L_{2+}} \wedge C_{R_{2+}} \wedge L_{2} \wedge R_{2}\right)=1 .
$$

From this equality and Hardy relation D4, a contradiction is derived.

I would like to explain intuitively the structure of the above proof, ignoring the details. Let us represent (9) and (10) respectively as follows:

$$
\begin{aligned}
& {\left[L_{1+} \Rightarrow C_{R_{2+}}\right]_{L_{1}},} \\
& {\left[C_{R_{2+}} \Rightarrow R_{2+}\right]_{R_{2}},}
\end{aligned}
$$

where, the subscripts indicate measurement settings. Similarly, (11) and (12) are represented respectively as follows:

$$
\begin{aligned}
& {\left[R_{1+} \Rightarrow C_{L_{2+}}\right]_{R_{1}},} \\
& {\left[C_{L_{2+}} \Rightarrow L_{2+}\right]_{L_{2}} .}
\end{aligned}
$$


Then, although not strict, by (19) and (21), we have

$$
\left[L_{1+} \wedge R_{1+} \Rightarrow C_{L_{2+}} \wedge C_{R_{2+}}\right]_{L_{1} \wedge R_{1}} .
$$

Also, by (20) and (22), we have

$$
\left[C_{L_{2+}} \wedge C_{R_{2+}} \Rightarrow L_{2+} \wedge R_{2+}\right]_{L_{2} \wedge R_{2}} .
$$

Using (23) and (24), contradiction is derived as follows. Suppose $\left[L_{1+} \wedge R_{1+}\right]_{L_{1} \wedge R_{1}}$ is true. This is possible because of Hardy relation D1. Then, using (23), $\left[C_{L_{2+}} \wedge C_{R_{2+}}\right]_{L_{1} \wedge R_{1}}$ is true. Because of $\mathcal{C}$-independence, $\left[C_{L_{2+}} \wedge C_{R_{2+}}\right]_{L_{2} \wedge R_{2}}$ is true. Then, $\left[L_{2+} \wedge R_{2+}\right]_{L_{2} \wedge R_{2}}$ becomes true by (24), which contradicts with Hardy relation D4.

\section{Discussion}

As a consequence of the mathematical fact (Fact 4) which proved by Graßhoff et al., we showed there exists no common cause model that satisfies B1 and $\mathcal{C}$-independence when a composite system is in a maximally entangled state (Fact 5). Also, in this paper, we proved that there exists no common cause model satisfying $\mathrm{B} 1$ and $\mathcal{C}$-independence when a composite system is in any non-maximally entangled state (Fact 6). Therefore, it became clear that for any entangled state, there exists no common cause model satisfying B1 and $\mathcal{C}$-independence.

If the non-existence of a common cause model is limited to some particular quantum states, we could think that counterparts of such states do not exist in our future physical theory and it would be possible to have hope for the common-causal explanation. However, there exists no common cause model satisfying the locality requirements, not only when the physical system is in a particular state (i.e. a maximally entangled state like the singlet state). In any entangled state, such a model does not exist.

On the other hand, if we weaken $\mathcal{C}$-independence to B2, as mentioned in Fact 3 , for any quantum theoretical state we can construct a common cause model. Therefore, the author thinks that $\mathrm{B} 1$ and $\mathcal{C}$-independence is the limit point to derive inconsistency with quantum mechanical predictions.

The proof method of Fact 6 is similar to the method when Graßhoff et al. showed Fact 4, but there is also an important difference. As mentioned in the next paragraph of Fact 4, when two events are perfectly correlated, for the screening-off factors of the correlation, the determinism holds. Using this fact, Graßhoff et al. showed Fact 4 . They assumed the existence of three pairs of observables with perfect correlation and derived the determinism for each perfect correlation. When the determinism holds, common screening-off factors can be constructed for multiple correlations, therefore Bell's inequality is derived. ${ }^{8}$

On the other hand, the correlations $\operatorname{Corr}\left(L_{1+}, R_{2+}\right)$ and $\operatorname{Corr}\left(L_{2+}, R_{1+}\right)$ in Fact 6 are not perfect correlations. Taking the correlation $\operatorname{Corr}\left(L_{1+}, R_{2+}\right)$ as

\footnotetext{
${ }^{8}$ A similar proof can be seen in Higashi (2008).
} 
an example, when a composite system is in a non-maximally entangled state, $P\left(R_{2+} \mid L_{1+} \wedge L_{1} \wedge R_{2}\right)=1$ holds (i.e. D2), however, $P\left(L_{1+} \mid R_{2+} \wedge L_{1} \wedge R_{2}\right)=1$ does not hold. Both equations are needed to derive the determinism. Indeed, it is possible to construct a probability space as a counterexample. As shown in the proof of Fact 6 , we can derive $P\left(R_{2+} \mid C_{k}^{12} \wedge R_{2}\right)=1$ for some $k \in K_{12}$ from D2. However, we cannot derive $P\left(R_{2+} \mid C_{k}^{12} \wedge R_{2}\right)=0$ or 1 for any $k \in K_{12}$. For the correlations $\operatorname{Corr}\left(L_{1+}, R_{2+}\right)$ and $\operatorname{Corr}\left(L_{2+}, R_{1+}\right)$, the determinism does not hold. Thus, we cannot construct common screening-off factors for these correlations (at least in Graßhoff et al.'s way).

Also, note that what is shown in this paper is a limitation of common cause approaches in a classical probability spaces. We do not discuss any common cause approach in a quantum probability space. When considering the common cause of each correlation in a classical probability space, common cause events are commutative. Therefore, we have to think about the truth value of the propositions such as conjunction, disjunction, and etc of them. On the other hand, in a quantum probability space, common cause events are not necessarily commutative, thus in such a case, we have not to think about their truth value simultaneously. However, when considering common cause approaches in a quantum probability space, we will face another problem of how to interpret the propositions of non-commutative observables. We will consider this problem on another occasion.

\section{References}

[Aspect, Dalibard, \& Roger (1982)] Aspect, A., Dalibard, J., \& Roger, G. (1982). Experimental test of Bell's inequalities using time-varying analyzers. Physical Review Letters 49, 1804-1807.

[Bana, \& Durt (1997)] Bana, G., \& Durt, T. (1997). Proof of Kolmogorovian censorship. Foundations of Physics 27(10), 1355-1373.

[Bub (1997)] Bub, J. (1997). Interpreting the quantum world. Cambridge: Cambridge University Press, (Section 2.3).

[Graßhoff, Portmann, \& Wüthrich (2005)] Graßhoff, G., Portmann, S., \& Wüthrich, A. (2005). Minimal assumption derivation of a Bell-type inequality. The British Journal for The Philosophy of Science 56, 663-680.

[Hardy (1993)] Hardy, L. (1993). Nonlocality for two particles without inequalities for almost all entangled states. Physical Review Letters 71, 1665-1668.

[Higashi (2008)] Higashi, K. (2008). The limits of common cause approach to EPR correlation. Foundations of Physics 38, 591-609.

[Hofer-Szabó, Rédei, \& Szabó (1999)] Hofer-Szabó, G., Rédei, M., \& Szabó, L. E. (1999). On Reichenbach's common cause principle and Reichenbach's notion of common cause. The British Journal for the Philosophy of Science 50, 377-399. 
[Hofer-Szabó, Rédei, \& Szabó (2002)] Hofer-Szabó, G., Rédei, M., \& Szabó, L. E. (2002). Common-causes are not common common-causes. Philosophy of Science 69, 623-636.

[Hofer-Szabó (2011)] Hofer-Szabó, G. (2011). Bell $(\delta)$ inequalities derived from separate common causal explanation of almost perfect EPR anticorrelations. Foundations of Physics 41(8), 1398-1413.

[Nielsen, \& Chuang (2000)] Nielsen, M., \& Chuang, I. (2000). Quantum computation and quantum information. Cambridge: Cambridge University Press, (Section 2.5).

[Portmann, \& Wüthrich (2007)] Portmann, S., \& Wüthrich, A. (2007). Minimal assumption derivation of a weak Clauser-Horne inequality. Studies in History and Philosophy of Modern Physics, 38(4), 844-862.

[Redhead (1987)] Redhead, M. (1987). Incompleteness, nonlocality, and realism. Oxford: Oxford University Press, (Section 4.4).

[Reichenbach (1956)] Reichenbach, H. (1956). The direction of time. New York: Dover Publications.)

[Szabó (1998)] Szabó, L. E. (1998). On an attempt to resolve the EPR-Bell paradox via Reichenbachian concept of common cause. https://arxiv.org/abs/quant-ph/9806074/Accessed 6 July 2018.

[Szabó (2000)] Szabó, L. E. (2000). Attempt to resolve the EPR-Bell paradox via Reichenbach's concept of common cause. International Journal of Theoretical Physics 39, 901-911.

[Uffink (1999)] Uffink, J. (1999). The principle of the common cause faces The Bernstein paradox. Philosophy of Science 66, S512-S525.

[Van Fraassen (1982)] Van Fraassen, B. C. (1982). The charybdis of realism: Epistemological implications of Bell's inequality. Synthese 52, 25-38.

[Wigner (1970)] Wigner, E. P. (1970). On hidden variables and quantum mechanical probabilities. American Journal of Physics 38, 1005-1009. 